\section{The p400 E1A-associated protein is a novel component of the p53 $\rightarrow$ p 21 senescence pathway}

Ho Man Chan, ${ }^{1}$ Masako Narita, ${ }^{2}$ Scott W. Lowe, ${ }^{2}$ and David M. Livingston ${ }^{1,3}$

${ }^{1}$ Dana-Farber Cancer Institute, Harvard Medical School, Boston Massachusetts 02115, USA; ${ }^{2}$ Cold Spring Harbor Laboratory, Cold Spring Harbor, New York 11724, USA

Adenovirus E1A-associated p400 belongs to the SWI2/ SNF2 family of chromatin remodeling proteins. Here, we report that $\mathrm{p} 400$ is a component of the p53$\mathrm{p} 21^{\mathrm{WAF} 1 / \mathrm{CIP} 1 / \mathrm{sid} 1}$ pathway, regulating the $p 21$ transcription and senescence induction program. Acute depletion of p400 expression by shRNA (short hairpin RNA) synthesis led to premature senescence of untransformed human fibroblasts, whose features include G1 arrest, p21 induction, senescence-associated heterochromatic foci (SAHF), and $\beta$-gal staining. Importantly, p400shRNA-induced premature senescence phenotypes were rescued by coexpression of p53-shRNA or p21shRNA. Furthermore, p400 complex colocalized with p53 on the p21 promoter. These data suggest that the p400 complex inhibits p53 $\rightarrow$ p21 transcription and the development of premature senescence.

Supplemental material is available at http://www.genesdev.org.

Received June 22, 2004; revised version accepted November 19, 2004.

The N-terminal region of adenoviral E1A interacts with p400, a member of the SWI2/SNF2 chromatin remodeling family (Fuchs et al. 2001). E1A mutants defective in interacting with p400 cannot transform primary cells efficiently when coexpressed with activated Ras (Deleu et al. 2001; Fuchs et al. 2001; Seger et al. 2002), suggesting that $\mathrm{p} 400 / \mathrm{E} 1 \mathrm{~A}$ complexes participate in the transformation process. p400 interacts with SV40 large T antigen (Lill et al. 1997b), c-Myc (Fuchs et al. 2001), and the tumor suppressor, p53 (Lill et al. 1997a). It is also a component of the human TIP60/NuA4 complex (Ikura et al. 2000; Fuchs et al. 2001; Cai et al. 2003; Doyon et al. 2004), which has specific histone H4/H2A acetyltransferase activity (Ikura et al. 2000; Doyon et al. 2004).

ChIP (chromatin immunoprecipitation) analysis has detected p400 complexes at certain c-Myc and E2F-regulated promoters (Frank et al. 2003; Taubert et al. 2004). Their recruitment to such promoters correlated with an increase in histone acetylation in the surrounding chromatin and induction of transcription. These findings

[Keywords: p400; senescence; p53; p21; E1A; myc]

${ }^{3}$ Corresponding author.

E-MAIL david Livingston@dfci.harvard.edu; FAX (617) 632-4381.

Article and publication are at http://www.genesdev.org/cgi/doi/10.1101/ gad.1280205. suggest that p400-containing complexes exert key chromatin-remodeling functions and participate in transcription regulation.

In the present study, retroviral-mediated shRNA was used to target p400 complexes and to investigate the outcomes of this perturbation in human cells. Specifically, acute depletion of p400 induced premature senescence in multiple, untransformed human fibroblasts. p400 shRNA-induced SAHF and other premature senescence phenotypes were rescued by coexpression of shRNA for $\mathrm{p} 53$ or $\mathrm{p} 21$, but not $\mathrm{pRb}$. p400-depleted cells revealed elevated p21 mRNA and protein expression. In keeping with these findings, p400 was detected at or near the p53-binding site in the $p 21$ promoter. These data suggest that p400 complexes inhibit p53 $\rightarrow$ p21 expression, and that deregulated p21 expression leads to senescence.

\section{Results and Discussion}

p400-shRNAs cause cell cycle arrest, SAHF, and premature senescence in untransformed human fibroblasts

Multiple retroviral vectors each encoding an shRNA directed against human $\mathrm{p} 400$ were constructed. The program for antibiotic selection and analysis of cells expressing these vectors is shown in Figure 1A. When IMR90 primary human diploid fibroblasts were transduced with shRNA-p400\#2 or shRNA-p400\#4, endogenous p400 protein expression was suppressed (Fig. 1B, cf. lanes 2,4 and 1). Moreover, shRNA-p400\#2 and ShRNA-p400\#4 effectively suppressed p400 expression in HeLa, T98G, telomerase-immortalized BJ fibroblasts (BJ-tert), U2OS, and a primary human lung fibroblast strain (Supplementary Fig. 7; data not shown). ShRNAp400\#3 did not significantly affect the p400 protein level (Fig. 1B) and was used as a negative control (Fig. 1D). Suppression of $\mathrm{p} 400$ expression was associated with G1 arrest (Fig. 1D, graph i), decreased BrdU incorporation (Fig. 1D, graph ii), and it inhibited proliferation of IMR90 (Fig. 1C). Similar results were observed in BJ-tert (data not shown). None of these effects was detected when experiments were performed on two transformed human cell lines in which p53 function is compromised, including HeLa and T98G (Supplementary Fig. 2).

Unlike naive IMR90, p400 shRNA-treated IMR90 became elongated and spindle shaped. When their DNAstaining properties were examined by fluorescence microscopy, $\sim 40 \%-60 \%$ of the culture revealed clustered DAPI-dense regions (Fig. 2A, panel 3). These structures were virtually absent in control cells (Fig. 2A, panel 1) and are decidedly unusual in human tissue culture cells in general. Spots of this type were recently described in IMR90 undergoing replicative senescence or experiencing activated Ras expression (Narita et al. 2003). They were referred to as senescence-associated heterochromatic foci (SAHF) (Narita et al. 2003). SAHF were also observed following p400 depletion in normal diploid lung fibroblasts and in telomerase-immortalized BJ fibroblasts (data not shown). In contrast, T98G and HeLa cells failed to display SAHF before or after p400 knockdown (Supplementary Fig. 2; data not shown). Where tested, the above-noted, DAPI-dense regions costained with an 
A

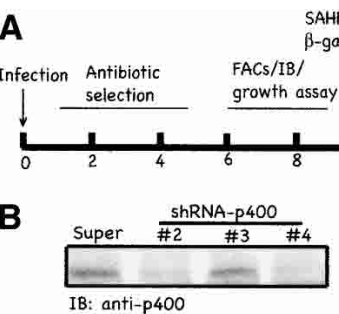

D i)

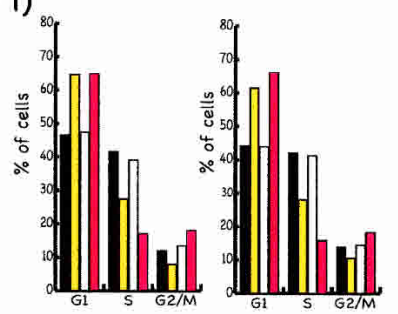

C

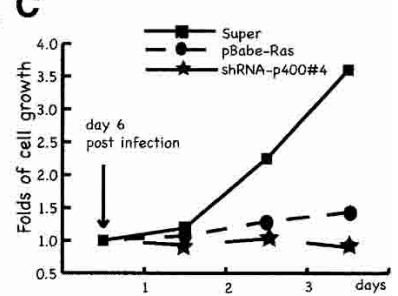

ii)

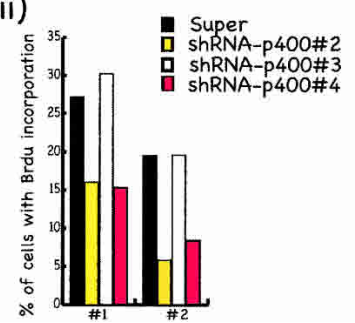

Figure 1. shRNA-p400-mediated cell cycle arrest in IMR90. $(A)$ Experimental design and time frame. $(B)$ Immunoblot of $\mathrm{p} 400$ from IMR90. (Super) The empty retroviral backbone control. Total protein loaded was standardized by Bradford assay (Bio-Rad). (C) Cell growth assay performed in IMR90. $(D)$ FACS analysis and BrdU incorporation assay from two independent experiments.

antibody to methylated histone H3 Lys 9 (Fig. 2A, panel 4), consistent with earlier findings (Narita et al. 2003).

The development of a SAHF phenotype following p400 depletion led to a further analysis of whether p400 shRNA-treated cells, like their activated Ras-producing counterparts, enter premature senescence. Indeed, p400 shRNA-treated IMR90 (Fig. 2B) displayed senescent $\beta$-gal staining, again suggesting that they were senescent. Two independent shRNAs designed against two, different sequences of p400 suppressed p400 protein expression and cell cycle progression (Fig. 1D). Each also induced SAHF (Fig. 2A, panel 3 for shRNA-p400\#4; data not shown) and senescent $\beta$-gal staining (Fig. $2 B$ ), linking this phenotype to the specific targeting of $\mathrm{p} 400$.

To determine whether knockdown of other components of the p400 complex lead to similar phenotypes, shRNAs were designed against TIP48 and TIP49, two subunits of this complex (Fuchs et al. 2001). Depletion of TIP48 or TIP49 expression by shRNA recapitulated the phenotypes of shRNA-p400 in IMR90 and BJ-tert, including G1 arrest, decreased BrdU incorporation, and the development of SAHF (Supplementary Fig. 1). Taken together, these data imply that acute loss-of-function of the p400 complex is associated with premature senescence.

Thus far, we have not detected a spontaneous decrease of expression of the p400 complex in cells undergoing replicative senescence (data not shown). If a perturbation of p400-complex function is required for this outcome, it must involve other molecular alteration(s) than simple protein depletion.

\section{shp400-infected IMR90 has elevated p21 expression}

Since oncogenic Ras overexpression and p400 depletion both induced premature senescence and SAHF (Serrano et al. 1997; Narita et al. 2003), we compared the molecular features of these two states in search of similarities

and differences. Both perturbations led to maximum senescent $\beta$-gal staining at approximately day 10 post-infection. Activated Ras overexpression had no effect on p400 protein level, and shRNA-p400 transduction did not activate endogenous Ras expression (Fig. 2C). Moreover, in Ras-expressing cells, there was up-regulation of p16 (Fig. 2C), and some enhancement of p53 Ser15 phosphorylation (Fig. 2C), consistent with previous reports (Serrano et al. 1997; Ferbeyre et al. 2000; Narita et al. 2003). However, shRNA-p400 did not affect the p16 protein level or p53 Ser15 phosphorylation, but was associated with p21 up-regulation (Figs. 2C, 3B). By quantitative RT-PCR, significant increases in the p21 mRNA level were reproducibly detected in shRNA-p400-treated cells (Fig. 2D), suggesting an enhancement of p21 transcription.

In keeping with its function as a cdk inhibitor (Sherr and Roberts 1999), p21 (also known as sdi [senescent cell-derived inhibitor]) is highly expressed in senescent cells and inhibits DNA synthesis (Noda et al. 1994). Indeed, it can elicit this phenotype on its own, for overexpression of p21 can result in premature senescence (McConnell et al. 1998) and SAHF (M. Narita and S.W. Lowe, unpubl.). Moreover, it serves as a lifespan regulator, because knocking out p21 can extend the lifespan of human fibroblasts (Brown et al. 1997).

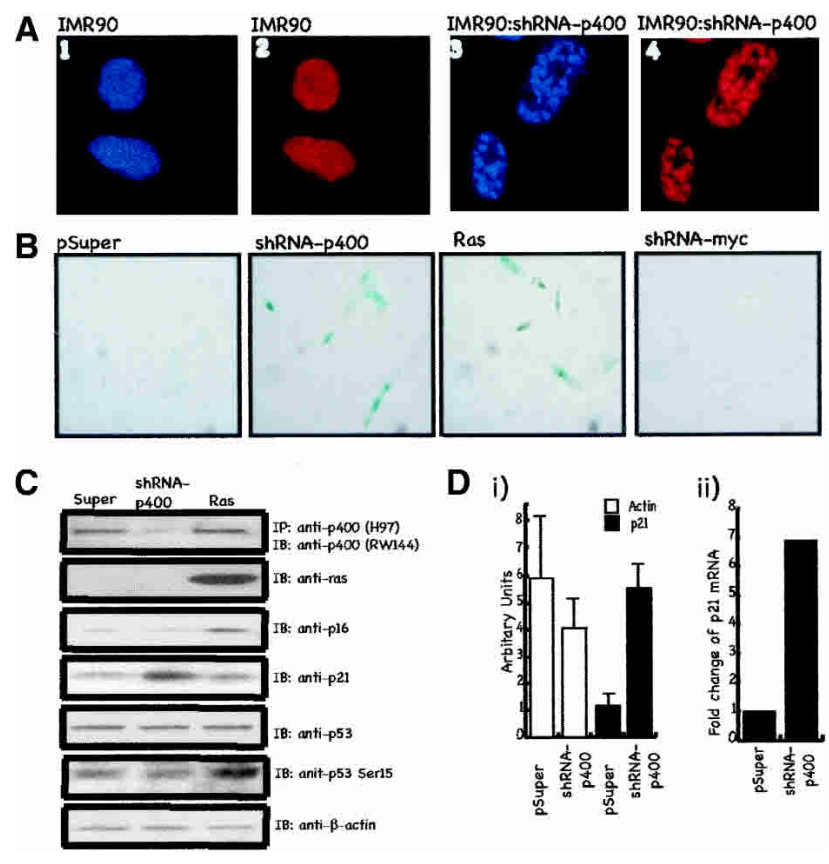

Figure 2. shRNA-p400 induces SAHF and premature senescence in IMR90. (A) DNA staining by DAPI (panels 1,3) and H3K9-Me staining (panels 2,4) of cells as indicated. The cells in panels 2 and 4 correspond to the cells in panels 1 and 3, respectively. $(B)$ Senescent $\beta$-gal staining of IMR90 following the indicated perturbations. Ras was included as a positive control, and shRNA-c-myc and pSuper were included as negative controls. $(C)$ IB and IP-IB of the indicated proteins from IMR90 exposed to the indicated perturbations. $(D)$ Quantitative RT-PCR measuring p21 mRNA in control IMR90 or IMR90 exposed to shRNA against p400. Unnormalized actin and p21 mRNA levels are presented in graph i. (Graph ii) After normalization against actin mRNA, the relative p 21 mRNA abundance was calculated. The data presented are average values obtained from quadruplicate samples. 
Chan et al.

Inactivation of $p 53 / p 21$, but not $p R b$, rescues $p 400$ shRNA-induced premature senescence in IMR90

Ras-induced SAHF can be partially rescued by suppressing the $\mathrm{pRb} / \mathrm{E} 2 \mathrm{~F}$ pathway (Narita et al. 2003), while inactivation of p53 had no such effect (Narita et al. 2003). In light of these findings, we asked whether the $\mathrm{pRb}$ or p53 pathways contribute to shRNA p400-induced premature senescence. Consistent with a previous report (Narita et al. 2003) and as a positive control, it was possible to use shRNA-pRb to partially rescue Ras-induced SAHF (data not shown). However, shRNA-pRb had no effect on shRNA-p400-induced SAHF, cell cycle arrest, or senescent $\beta$-gal staining (Fig. 3A, Exp1; Supplementary Fig. 3). Its knockdown of $\mathrm{pRb}$ expression is shown in Supplementary Figure 4A. Furthermore, overexpression of cyclin E or cdk4 (R24C) (Supplementary Fig. 4B), both predicted to relieve pocket protein-mediated transcription repression of certain E2F-activated genes (Rane et al. 2002), were also unable to rescue shRNA-p400-induced SAHF and senescence $\beta$-gal staining (Fig. 3A, Exp4; Supplementary Fig. 3). In contrast, shRNA depletion of p53 did rescue shRNA-p400-induced SAHF, cell cycle arrest, and senescent $\beta$-gal staining (Fig. 3A, Exp2 and Exp4; Supplementary Figs. 3, 5). Importantly, expression of shRNA-p53 did not reduce the efficiency with which shRNA-p400 suppressed p400 expression in cells exposed to a combination of shRNAs for p53 and for p400 (Fig. 3B). Furthermore, overexpression of a dominantnegative p53 mutant (p53DD) partially suppressed shRNA-p400-induced SAHF (Fig. 3A, Exp5). Taken together, the above-noted data imply that the SAHF and senescence effects associated with p400 knockdown depend upon the existence of normal p53, but not $\mathrm{pRb}$, function. This is the opposite of the mechanism driving SAHF that arise after activated Ras expression (Narita et al. 2003).

While p400 depletion led to p21 up-regulation, p53 depletion completely abolished basal p21 expression (Fig. 3B). The latter effect is likely dominant, because the p21 level remained undetectable in cells treated with both shRNA-p53 and shRNA-p400 (Fig. 3B). One possible prediction from these and the above-noted results is that $\mathrm{p} 21$ is the prime lifespan control protein at work in the p400 pathway.
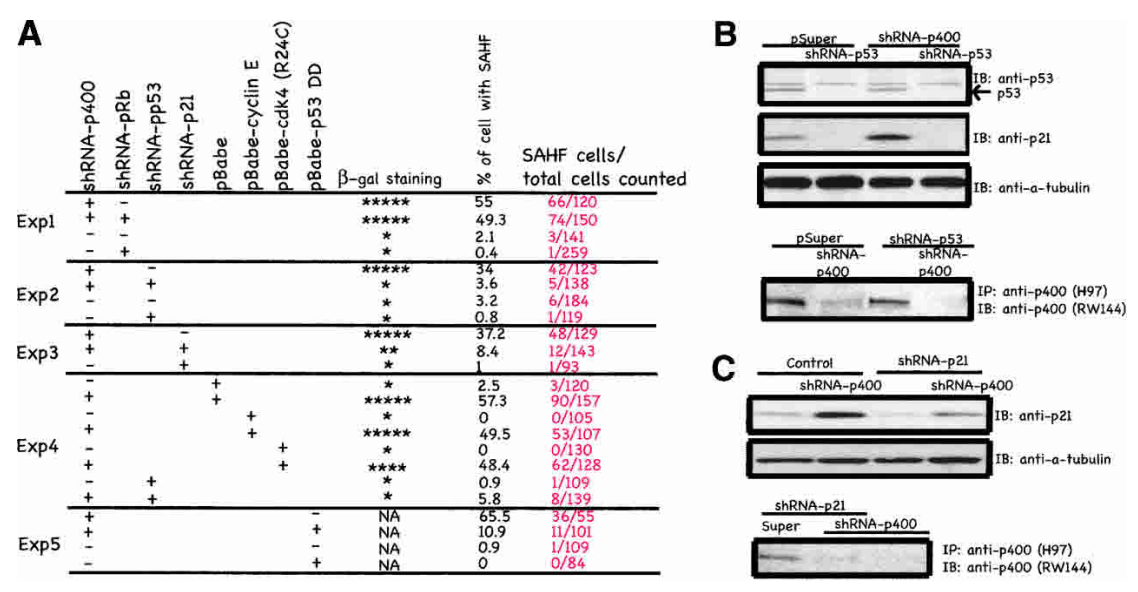

Figure 3. Inactivation of $\mathrm{p} 53 / \mathrm{p} 21$, but not pocket family proteins, rescues shRNA-p400mediated premature senescence. (A) Summary of SAHF and senescent $\beta$-gal staining in IMR90 exposed to the indicated perturbations. $(B, C)$ IB and IP-IB of the indicated IMR90 proteins after the indicated perturbations.
To test this, we asked whether $\mathrm{p} 21$ depletion also rescues cells from shRNA-p400-mediated premature senescence. Down-regulation of $\mathrm{p} 21$ expression by shRNAp21 (Fig. 3C, top, cf. lanes 1 and 3, and lanes 2 and 4) substantially reduced the incidence of shRNA-p400-induced senescent phenotypes (Fig. 3A, Exp3). A large fraction of cells treated with shRNA-p400 revealed SAHF and strong p21 staining (for examples, cf. Supplementary Fig. 6). Both SAHF and p21 staining were greatly reduced in cells doubly treated with shRNA-p21 and shRNAp400 (Supplementary Fig. 6). Importantly, the shRNA p21 reagent did not overtly compromise the ability of shRNA p400 to suppress p400 expression in the cells doubly infected with viruses encoding shRNAs for $\mathrm{p} 21$ and p400 (Fig. 3C, bottom). Overall, we conclude that the p53-p21 pathway contributes to shRNA-p400-induced premature senescence.

It is understandable that no phenotype was observed when shRNA-p400 was introduced into HeLa and T98G cells (Supplementary Fig. 2), since p53 function in these cells is compromised by the presence of HPV E6 and of a genomic loss of function mutation, respectively (Hall et al. 1998; Goodwin and DiMaio 2001). To further test the above-noted hypothesis, shRNA-p400 was transduced into U2OS cells, an osteosarcoma cell line carrying wildtype $p 53$ alleles. In p400-depleted U2OS cells, increased p21 expression and G1-arrested cells were observed (Supplementary Fig. 7A,B). Consistent with the observation in IMR90 (Fig. 2C), p400 depletion in U2OS cells did not affect the p53 expression level (Supplementary Fig. 7C). Unlike IMR90, however, SAHF were not observed in U2OS following p400 depletion, or exogenous p21 overexpression (data not shown). Therefore, elevated expression of $\mathrm{p} 21$ is not sufficient to cause SAHF or premature senescence in U2OS. This notwithstanding, p21 overexpression or p400 depletion were not inert events in these cells, since both led to marked inhibition of DNA synthesis (Supplementary Fig. 7D).

\section{p400 complexes colocalize with p53 on the p21 promoter}

p21 expression is normally up-regulated by $\mathrm{p} 53$. One outcome is G1 arrest (el-Deiry et al. 1993; Macleod et al. 1995; Vogelstein et al. 2000). Given that p400 depletion induces p21 synthesis, one wonders whether p400 plays a direct role in down-regulating p53-dependent transcription of $p 21$. In this regard, using ChIP analysis, we detected p400 bound to a region that overlaps the distal p53-binding site within the $p 21$ promoter (Fig. 4B, \#1, cf. lanes 8 and 3,7). Consistently, TIP49, a p400 complex member (Fuchs et al. 2001), was also detected in the same region (Fig. 4C). p400 binding at this site was specific, since no p400 was detected at the proximal TGF $\beta$ response element-containing region within the p21 promoter (Fig. 4B, \#2), nor was it bound to a region $\sim 3 \mathrm{kbp}$ downstream of the $p 21$ transcription initiation site (Fig. 4B, \#3).

By performing ChIP with an anti- 
p400 Ab and then reimmunoprecipitating with anti-p53 $\mathrm{Ab}$ (DO-1), a PCR-amplified signal was detected from the distal p53-binding region (Fig. 4D, \#1), but not from the region $3 \mathrm{kbp}$ downstream of the transcription initiation site (Fig. 4D, \#3). Therefore, both p53 and p400 were colocated within the distal p53-binding/regulatory region of the $p 21$ promoter. These data are consistent with a model in which interplay between p53 and p400 contributes to normal regulation of the $p 21$ promoter. While p53 exerts a positive effect on this element, p400 appears to exert a negative one. As a result, depletion of $\mathrm{p} 400$ elicited p53-dependent transcription of the $p 21$ gene, and elevated p21 expression can lead to premature senescence (McConnell et al. 1998).

By quantitative ChIP analysis, multiple experiments demonstrated $60 \%-70 \%$ more p53 bound to site \#1 in IMR90 cells depleted of p400, compared with control IMR90-pSuper cells (Fig. 4E). This phenomenon is specific to the $p 21$ promoter, since there was no increase in

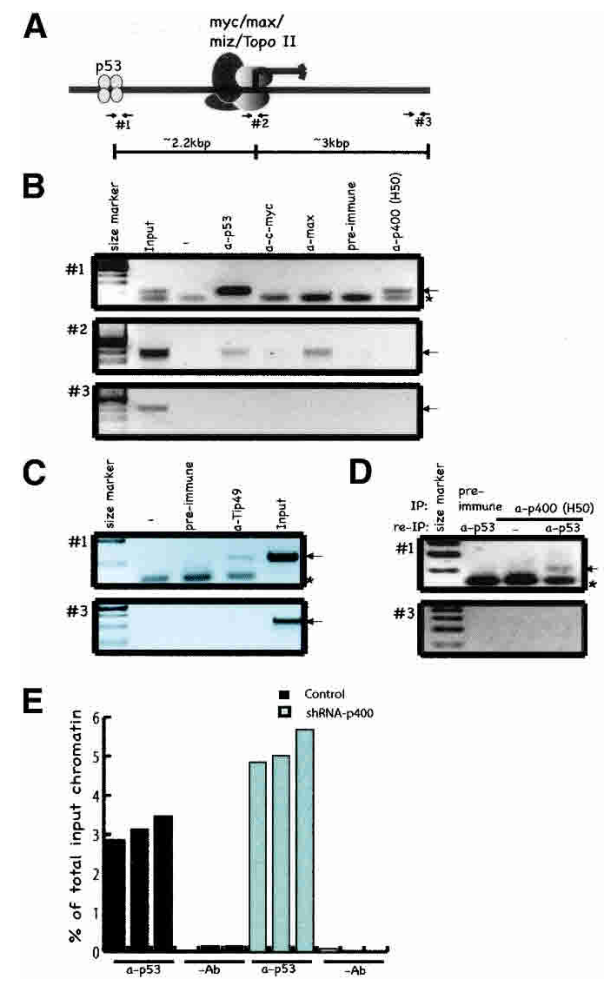

Figure 4. p400 colocalizes with p53 on the $p 21$ promoter. (A) Diagrammatic presentation of the $p 21$ promoter, indicating the primers used for ChIP (\#1, \#2, and \#3). (B-D) ChIP analysis of p400 complexes bound at the $p 21$ promoter using the indicated primer sets. Antibodies used for ChIP are indicated. Minus sign (-) indicates a control reaction mixture with no $\mathrm{Ab}$ added. $\mathrm{p} 53$ and max bound to regions \#1 and \#2, respectively, were as reported (Kaeser and Iggo 2002; Seoane et al. 2002; Wu et al. 2003). The presence of p53 in \#2 could be consistent with the report of a second p53-binding site $\sim 1$ $\mathrm{kbp}$ upstream of the transcription start site in the $p 21$ promoter (Resnick-Silverman et al. 1998). The arrows indicate the specific PCR products, and the asterisk indicates the position of a primer dimmer band. $(E)$ Quantitative analysis of p53 binding to the $p 21$ promoter in control IMR90 and IMR90 depleted of p400. Results from three different ChIP experiments are presented. In each ChIP experiment, triplicate samples were subjected to quantitative PCR, and the average value was used to calculate percent of total input chromatin bound. In three experiments, there were, respectively, $1.69,1.60$, and 1.64 times more p53 binding to \#1 in IMR90-shRNAp400 cells compared with control cells.

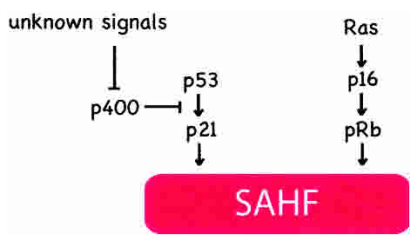

Figure 5. A model of $\mathrm{p} 400$ regulation of $\mathrm{p} 53 \rightarrow \mathrm{p} 21$ expression and its related senescence program. p400-p53/p21 and Ras-p16/pRb are two distinct pathways leading to SAHF. p400 represses p53 $\rightarrow$ p2 1 transcription to prevent premature senescence. It remains to be determined whether $\mathrm{p} 400$ complex function is perturbed in replicative senescence, and if so, whether its perturbation contributes to the biological outcome.

p53 binding to the puma or bax promoters in the same experiments (data not shown). Furthermore, no p400 was detected at the p53-binding region of the puma or bax promoters (data not shown). Thus, it is possible that increased p53 binding to the $p 21$ promoter in p400-depleted IMR90 contributed to the enhanced p21 expression observed.

In conclusion, p400, an SWI2/SNF2 chromatin-remodeling factor, plays a role in regulating p53 $\rightarrow$ p21 transcription and eliciting a cellular senescence program. Acute depletion of p400 or components of the p400 core complex in primary cells led to G1 arrest, SAHF, induction of $p 21$, and senescence $\beta$-gal staining, all features indicative of cells entering senescence (Narita et al. 2003). By epistatic analysis, p400 shRNA-mediated senescence has been linked to the p53/p21 pathway, but not the $\mathrm{pRb}$ pathway. This is the opposite of the mechanism leading to Ras-induced SAHF (Narita et al. 2003). These findings elicit speculation that $p 21$ and $p 16$ participate in distinct senescence pathways that respond to different cues (Fig. 5; Herbig et al. 2004).

Cellular senescence has been hypothesized to serve as a barrier against tumor development. The ability to overcome senescence is a prerequisite for tumor formation (Sager 1991; Hanahan and Weinberg 2000). We have observed that both E1A and c-Myc up-regulate p400 expression (data not shown). Furthermore, p400 is required for E1A-dependent apoptosis (A. Samuelson, M. Narita, and S.W. Lowe, unpubl.). Given the results reported above, one wonders whether E1A or c-Myc, by up-regulating p400 expression, override p53/p21-dependent cell cycle arrest, an outcome that was suggested to sensitize cells to p53-dependent apoptosis (Seoane et al. 2002). If so, one would argue that c-Myc and/or E1A do not inactivate p400. Rather, one wonders whether c-Myc/p400 or E1A/ p400 complexes exhibit a qualitative alteration in p400 function or even its activation. Since senescence is suspected of serving as a major component of tumor suppression and is associated, in at least one setting, with suppression of p400 complex function, it is conceivable that up-regulation of $\mathrm{p} 400$ expression/function by certain oncogenes (and, quite possibly, its binding to the $\mathrm{N}$-terminal region of E1A [Fuchs et al. 2001]) contributes to neoplastic transformation. An analysis of whether p400 function is deregulated in certain human tumors now seems timely.

\section{Materials and methods}

Cell culture, cell cycle analysis, and plasmids

All cells were cultivated at $37^{\circ} \mathrm{C}$ in a $10 \% \mathrm{CO}^{2}$-containing atmosphere; 293T, IMR90, HeLa, U2Os, and T98G were maintained in DMEM 
supplemented with $10 \%$ FBS and antibiotics. BJ-Tert cells were cultured in 4:1 DMEM/medium 199 (Invitrogen), supplemented with 15\% FBS and antibiotics. Retroviral production and infection protocols were as described (Serrano et al. 1997; Narita et al. 2003). When cells were doubly infected by viruses, cells were first selected with Puromycin $(2 \mu \mathrm{g} / \mathrm{mL})$ for $2 \mathrm{~d}$, followed by Hygromycin $(75 \mu \mathrm{g} / \mathrm{mL})$ for $2 \mathrm{~d}$. Routinely, $60 \%-80 \%$ of the cells survived selection. The cell growth assay and senescence $\beta$-gal-staining protocol have been described (Serrano et al. 1997; Narita et al. 2003). For FACS and BrdU incorporation assays, $4 \times 10^{5}$ cells were seeded on 10-cm plates $30-36 \mathrm{~h}$ before harvesting. Ten micromolar of BrdU was added to the culture medium for 45 min before harvesting. BrdU-free cells (and stained with $\alpha$-BrdU Ab) were included as negative controls. The following virus combinations were used in the experiment presented in Figure 3, Expl [pSuper-shRNA-Rb(Hygro) + pSuper-shRNAp400\#3(Puro); pSuper-shRNA-Rb(Hygro) + pSuper-shRNA-p400\#4(Puro); pSuper(Hygro) + pSuper-shRNA-p400\#3; pSuper(Hygro) + pSuper-shRNAp400\#4(Puro)]. In Exp2, [pSuper-shRNA-p400\#4(Hygro) + pSuper(Puro); pSuper-shRNA-p400\#4(Hygro) + pSuper-shRNA-p53(Puro); pSuper(Hygro) + pSuper(Puro); pSuper(Hygro) + pSuper-shRNA-p53(Puro)]. In Exp3, [pSuper (Puro) + pSuper-shRNA-p400\#4(Hygro); pSuper-shRNA-p21(Puro) + pSupershRNA-p400\#4(Hygro) and pSuper-shRNA-p21(Puro) + pSuper(Hygro)]. In Exp5, [pSuper(Puro) + pBabe(neo); pSuper-shRNA-p400(Puro) + pBabe(neo); pSuper(Puro) + pBabe-p53DD(neo); pSuper-shRNA-p400(Puro) + pBabep53DD(neo)]. pBabe (Puro)-cyclin E, pBabe (Puro)-cdk4 (R24C) and pBabe (Puro)-RasV12 have been described (Serrano et al. 1997). pBabe-p53DD (Shaulian et al. 1992) was a kind gift of Dr. W. Hahn (Dana-Farber Cancer Institute, Boston, MA). shRNAs against p53 and pRb were described in Voorhoeve and Agami (2003). shRNAs against p400, and TIP49 were cloned into pSuperRetro-(Puro) or pSuperRetro-(Hygro)-based vectors (Oligoengine). Sequence information can be obtained upon request.

\section{Immunofluorescence (IF)}

Cells were fixed with $4 \%$ paraformaldehyde and subjected to DAPI staining. The IF procedure for anti-dimethyl H3K9 (Upstate Biotechnology) and p21 (c-19, Santa Cruz) Ab was described previously (Ganesan et al. 2002).

\section{Antibodies}

Antibodies against c-myc (N262), max (c-17), pRb (c-15), and p21 (c-19), were from Santa Cruz Biotechnology. Antibody against $\alpha$-tubulin (T5168) was from Sigma. Antibody against pRb (G3-245) and the BrdU-staining kit were from Pharmingen. Antibodies against pl6 (05-418) and antiH3K9Me were from Upstate Biotechnology. p53 Ab (DO-1) was from Calibiochem. p53 Ser15 Ab and anti-p21 (DCS60) were from Signal Transduction Lab. Antibodies against TIP49 and TIP48 were raised as rabbit polyclonal sera against GST-TIP49 (full-length) and His-TIP48 (full-length), respectively. Antibodies against p400 (H97) and (H50) were raised against GST-p400 (1-300) and an N-terminal p400 peptide (SEGEEQPAHPNPPPS), respectively. Anti-p400 (RW144) was described (Fuchs et al. 2001). All polyclonal Abs were affinity purified.

\section{Immunoprecipitation (IP) and ChIP}

IP were performed by a slight modification of a prior protocol (Fuchs et al. 2001). Specifically, cell extract was collected in NETN buffer $150 \mathrm{mM}$ Tris- $\mathrm{HCl}$ at $\mathrm{pH} 8.0,150 \mathrm{mM} \mathrm{NaCl}, 0.1 \% \mathrm{NP}-40$ and protease inhibitor cocktail [Roche]), supplemented with $0.5 \%$ Triton. ChIP and IP-reIP ChIP were performed as described previously (Shang et al. 2000). Primers used in the ChIP assays were described (Kaeser and Iggo 2002; Wu et al. 2003). For quantitative ChIP analysis on the distal p53-binding site in the p21 promoter, the primers CCСТTCCTCACCTGAAAACA and GTG GCTCTGATTGGCTTTCTG were used. This set of primers did not yield primer dimers in PCR reactions. The method for quantitating the results of ChIP experiments has been described (Frank et al. 2001).

Quantitative RT-PCR

Total cellular RNA was harvested by RNeasy (QIAGEN). First-strand cDNA synthesis was carried out using the Superscript first-strand synthesis system (Invitrogen). Quadruplicate samples were subjected to quantitative PCR using iCycler (Bio-Rad), and the primers were described (Kaeser and Iggo 2002). PCR for actin mRNA was used as an internal control. The relative abundance of p21 mRNA was calculated after normalization using actin mRNA.

\section{Acknowledgments}

We thank all members of the Livingston and Nakatani labs for encouragement and helpful advice. In particular, we thank Drs. S. Ganesan, B. Xia, R. Drapkin, H. Tagami, and A. Kung. We also thank Ms. F. Zhang for help with ChIP, Dr. Q. Shi for RT-PCR, and Dr. M. Narita (Lowe lab) for technical assistance. In addition, we wish to extend our gratitude to Drs. J.J. Zhao, R. Agami, W. Hahn, J. Sedivy, G. Peters, B. Amati, and P. Andreassen for reagents, plasmids, cell lines, and helpful discussions. H.M.C. is funded by a Human Frontiers Long-term Fellowship. This work was funded by grants to D.L. from the National Cancer Institute.

\section{References}

Brown, J.P., Wei, W., and Sedivy, J.M. 1997. Bypass of senescence after disruption of p21CIP1/WAF1 gene in normal diploid human fibroblasts. Science 277: 831-834.

Cai, Y., Jin, J., Tomomori-Sato, C., Sato, S., Sorokina, I., Parmely, T.J., Conaway, R.C., and Conaway, J.W. 2003. Identification of new subunits of the multiprotein mammalian TRRAP/TIP60-containing histone acetyltransferase complex. J. Biol. Chem. 278: 42733-42736.

Deleu, L., Shellard, S., Alevizopoulos, K., Amati, B., and Land, H. 2001. Recruitment of TRRAP required for oncogenic transformation by E1A. Oncogene 20: 8270-8275.

Doyon, Y., Selleck, W., Lane, W.S., Tan, S., and Cote, J. 2004. Structural and functional conservation of the NuA4 histone acetyltransferase complex from yeast to humans. Mol. Cell. Biol. 24: 1884-1896.

el-Deiry, W.S., Tokino, T., Velculescu, V.E., Levy, D.B., Parsons, R., Trent, J.M., Lin, D., Mercer, W.E., Kinzler, K.W., and Vogelstein, B. 1993. WAF1, a potential mediator of p53 tumor suppression. Cell 75: 817-825.

Ferbeyre, G., de Stanchina, E., Querido, E., Baptiste, N., Prives, C., and Lowe, S.W. 2000. PML is induced by oncogenic ras and promotes premature senescence. Genes \& Dev. 14: 2015-2027.

Frank, S.R., Schroeder, M., Fernandez, P., Taubert, S., and Amati, B. 2001. Binding of c-Myc to chromatin mediates mitogen-induced acetylation of histone $\mathrm{H} 4$ and gene activation. Genes \& Dev. 15: 2069-2082.

Frank, S.R., Parisi, T., Taubert, S., Fernandez, P., Fuchs, M., Chan, H.M., Livingston, D.M., and Amati, B. 2003. MYC recruits the TIP60 histone acetyltransferase complex to chromatin. EMBO Rep. 4: 575580.

Fuchs, M., Gerber, J., Drapkin, R., Sif, S., Ikura, T., Ogryzko, V., Lane, W.S., Nakatani, Y., and Livingston, D.M. 2001. The p400 complex is an essential E1A transformation target. Cell 106: 297-307.

Ganesan, S., Silver, D.P., Greenberg, R.A., Avni, D., Drapkin, R., Miron, A., Mok, S.C., Randrianarison, V., Brodie, S., Salstrom, J., et al. 2002. BRCAl supports XIST RNA concentration on the inactive X chromosome. Cell 111: 393-405.

Goodwin, E.C. and DiMaio, D. 2001. Induced senescence in HeLa cervical carcinoma cells containing elevated telomerase activity and extended telomeres. Cell Growth Differ. 12: 525-534.

Hall, A.R., Dix, B.R., O'Carroll, S.J., and Braithwaite, A.W. 1998. p53dependent cell death/apoptosis is required for a productive adenovirus infection. Nat. Med. 4: 1068-1072.

Hanahan, D. and Weinberg, R.A. 2000. The hallmarks of cancer. Cell 100: $57-70$.

Herbig, U., Jobling, W.A., Chen, B.P., Chen, D.J., and Sedivy, J.M. 2004. Telomere shortening triggers senescence of human cells through a pathway involving ATM, p53, and p21(CIP1), but Not p16(INK4a). Mol. Cell 14: 501-513.

Ikura, T., Ogryzko, V.V., Grigoriev, M., Groisman, R., Wang, J., Horikoshi, M., Scully, R., Qin, J., and Nakatani, Y. 2000. Involvement of the TIP60 histone acetylase complex in DNA repair and apoptosis. Cell 102: $463-473$.

Kaeser, M.D. and Iggo, R.D. 2002. Chromatin immunoprecipitation analysis fails to support the latency model for regulation of p53 DNA binding activity in vivo. Proc. Natl. Acad. Sci. 99: 95-100.

Lill, N.L., Grossman, S.R., Ginsberg, D., DeCaprio, J., and Livingston, D.M. 1997a. Binding and modulation of p53 by p300/CBP coactivators. Nature 387: 823-827.

Lill, N.L., Tevethia, M.J., Eckner, R., Livingston, D.M., and Moditahedi, N. 1997b. p300 family members associate with the carboxyl terminus of simian virus 40 large tumor antigen. J. Virol. 71: 129-137.

Macleod, K.F., Sherry, N., Hannon, G., Beach, D., Tokino, T., Kinzler, K., 
Vogelstein, B., and Jacks, T. 1995. p53-dependent and independent expression of p21 during cell growth, differentiation, and DNA damage. Genes \& Dev. 9: 935-944.

McConnell, B.B., Starborg, M., Brookes, S., and Peters, G. 1998. Inhibitors of cyclin-dependent kinases induce features of replicative senescence in early passage human diploid fibroblasts. Curr. Biol. 8: 351-354.

Narita, M., Nunez, S., Heard, E., Narita, M., Lin, A.W., Hearn, S.A., Spector, D.L., Hannon, G.J., and Lowe, S.W. 2003. Rb-mediated heterochromatin formation and silencing of E2F target genes during cellular senescence. Cell 113: 703-716.

Noda, A., Ning, Y., Venable, S.F., Pereira-Smith, O.M., and Smith, J.R. 1994. Cloning of senescent cell-derived inhibitors of DNA synthesis using an expression screen. Exp. Cell. Res. 211: 90-98.

Rane, S.G., Cosenza, S.C., Mettus, R.V., and Reddy, E.P. 2002. Germ line transmission of the Cdk4(R24C) mutation facilitates tumorigenesis and escape from cellular senescence. Mol. Cell. Biol. 22: 644-656.

Resnick-Silverman, L., St Clair, S., Maurer, M., Zhao, K., and Manfredi, J.J. 1998. Identification of a novel class of genomic DNA-binding sites suggests a mechanism for selectivity in target gene activation by the tumor suppressor protein p53. Genes \& Dev. 12: 2102-2107.

Sager, R. 1991. Senescence as a mode of tumor suppression. Environ. Health Perspect 93: 59-62.

Seger, Y.R., Garcia-Cao, M., Piccinin, S., Cunsolo, C.L., Doglioni, C., Blasco, M.A., Hannon, G.J., and Maestro, R. 2002. Transformation of normal human cells in the absence of telomerase activation. Cancer Cell 2: 401-413.

Seoane, J., Le, H.V., and Massague, J. 2002. Myc suppression of the p21(Cip1) Cdk inhibitor influences the outcome of the p53 response to DNA damage. Nature 419: 729-734.

Serrano, M., Lin, A.W., McCurrach, M.E., Beach, D., and Lowe, S.W. 1997. Oncogenic ras provokes premature cell senescence associated with accumulation of p53 and p16INK4a. Cell 88: 593-602.

Shang, Y., Hu, X., DiRenzo, J., Lazar, M.A., and Brown, M. 2000. Cofactor dynamics and sufficiency in estrogen receptor-regulated transcription. Cell 103: 843-852.

Shaulian, E., Zauberman, A., Ginsberg, D., and Oren, M. 1992. Identification of a minimal transforming domain of p53: Negative dominance through abrogation of sequence-specific DNA binding. Mol. Cell. Biol. 12: 5581-5592.

Sherr, C.J. and Roberts, J.M. 1999. CDK inhibitors: Positive and negative regulators of G1-phase progression. Genes \& Dev. 13: 1501-1512.

Taubert, S., Gorrini, C., Frank, S.R., Parisi, T., Fuchs, M., Chan, H.M., Livingston, D.M., and Amati, B. 2004. E2F-dependent histone acetylation and recruitment of the Tip60 acetyltransferase complex to chromatin in late G(1). Mol. Cell. Biol. 24: 4546-4556.

Vogelstein, B., Lane, D., and Levine, A.J. 2000. Surfing the p53 network. Nature 408: 307-310.

Voorhoeve, P.M. and Agami, R. 2003. The tumor-suppressive functions of the human INK4A locus. Cancer Cell 4: 311-319.

Wu, S., Cetinkaya, C., Munoz-Alonso, M.J., von der Lehr, N., Bahram, F., Beuger, V., Eilers, M., Leon, J., and Larsson, L.G. 2003. Myc represses differentiation-induced p21CIP1 expression via Miz-1-dependent interaction with the p21 core promoter. Oncogene 22: 351-360. 


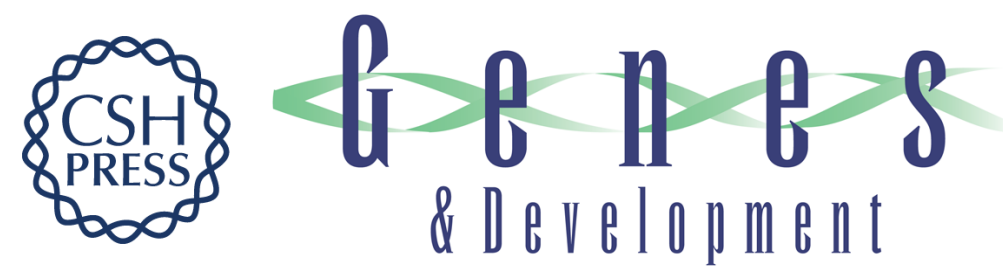

\section{The p400 E1A-associated protein is a novel component of the p53 $\rightarrow$ p21 senescence pathway}

Ho Man Chan, Masako Narita, Scott W. Lowe, et al.

Genes Dev. 2005, 19:

Access the most recent version at doi:10.1101/gad.1280205

\section{Supplemental http://genesdev.cshlp.org/content/suppl/2004/12/29/19.2.196.DC1 Material}

References This article cites 35 articles, 15 of which can be accessed free at: http://genesdev.cshlp.org/content/19/2/196.full.html\#ref-list-1

\section{License}

Email Alerting

Receive free email alerts when new articles cite this article - sign up in the box at the top Service right corner of the article or click here.

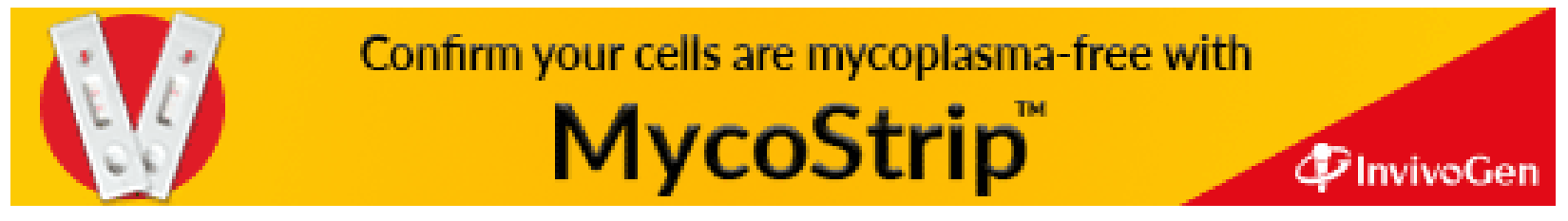

